\title{
LINGUISTIC ASPECTS OF CHARTED KNITTING PATTERNS
}

\author{
Baiba Egle ${ }^{1}$
}

\begin{abstract}
The paper outlines the linguistic and semiotic aspects of a specialty text - a knitting pattern. In today's busy world, nobody wants to read long and complicated texts if it can be easier illustrated by a photograph or a charted image that uses a set of specific symbols with their own, non-arbitrary meanings to make a text reader's time usage more effective but also clear. Aspects of signs, semiotics, and language games are also explored in alignment with knitting charts as the main reference, as a knitting chart symbol is a concrete referent in its context. Finally, the translation of knitting charts is described, including the importance of a chart key and what transformations of text have occurred in the translation of knitting charts from English into Latvian. Charted knitting patterns could become a that could be easily transferred between different languages and cultures, helping people share their skills and heritage. Knitting and linguistics and translation studies is a new area of research that could bring many new insights about crafting and specialized language use.
\end{abstract}

UDC Classification: 81’33, DOI: https://doi.org/10.12955/pss.v2.208

Keywords: knitting, charts, translation, symbol

\section{Introduction}

Everyone knows the old saying that a picture is worth a thousand words. Nobody wants to read long and complicated texts in today's busy world if it can be easier illustrated by a photograph or a graph. While everyone can explain what a photograph or a standard graph is, it is slightly more complicated to explain what a symbol is as there are many possible interpretations based on various contexts a symbol could be used in.

This paper aims at showing the complexity of symbol usage in knitting patterns and their linguistic aspects depending on previous knowledge of the text receiver and specialized background, as well as various aspects of chart translation as this problem has not yet been looked at in linguistics nor translation studies in any language pair.

Before the specifics of knitting patterns are looked at, one should think of the general description of symbols describes them as: "something that stands for or suggests something else by reason of relationship, association, convention, or accidental resemblance" (Merriam-Webster 2021). No matter their background or education level, many people would make a guess that a heart means love or affection. But what would " " mean to someone? Or perhaps, "-_"? Reasonably we might say that it wholly depends on the context and the person's knowledge. A coder might describe the various uses in their programming language; a linguist would have a different but equally correct description of it. There is a multitude of meanings possible, even down to no meaning at all, as language users automatically insert various marks as these in their text without thinking about what a backslash signifies from a grammatical standpoint.

A symbol is also "an arbitrary or conventional sign used in writing or printing relating to a particular field to represent operations, quantities, elements, relations, or qualities" (Merriam-Webster 2021). The two examples given in here are quite common, used in all kinds of texts. But in knitting, they do represent something else than what is their conventional meaning. Knitting charts and the symbols used within form an interconnected world of language and imagery, that is both universal (as anyone who knows the meaning of knitting chart symbols could theoretically read a chart and understand it no matter their native language or other languages the person knows) but also specific as cultural background and knitting traditions are known by the person.

While there are no mainstream dictionary definitions of what could be deemed as the only definition of a knitting chart, it can be simplified to:

a graphic representation, whereby the use of various symbols in the knitting process is explained without the use of words and shows complicated content in a compact and easily readable way. Each symbol used has a meaning, none are used arbitrarily.

This is the proposed definition of the term by the author of this paper that can be argued with, as especially knitters would have different opinions in the matter, as there are cultures which prefer to knit from charts (like Latvians, Estonians, also Lithuanians and many others) and other cultures (especially

\footnotetext{
${ }^{1}$ Ventspils University of Applied Sciences, Department of Translation Studies, Ventspils, Latvia. Baiba.egle@venta.lv
} 
North American) that vastly prefer the use of a written pattern. Knitting as a craft is both international and national. Therefore, opinions can differ, but many knitters would agree that in some ways, a charted pattern is more universal for many crafters, despite their background, for example, if one knows the 'language' of the knitting chart, one could easily use a Japanese knitting pattern without knowing any Japanese language aspects, such as vocabulary and grammar.

\section{Sign, Meaning, and Semiosis in knitting}

There are dozens of discussions online about how to read a charted knitting pattern, whether it is easy or not, whether it is better than a written pattern. Like about any matter with a large group of users, there will be plenty of differing opinions about what is best and what is not. Some of the discussion stems from the fact that some knitters/readers do not fully understand what a charted pattern is and how to use it.

A knitting chart symbol is also a sign from a semiotic point of view. "The thing to which a sign refers is known, logically, as the referent. There are two kinds of referents: (1) a concrete referent, such as the animal designated by the word cat, and (2) an abstract referent [...]" (Danesi, 2004, 5). Here we can see the two possible situations where a sign's meaning can be either concrete or not. In a knitting pattern, sometimes the situation can arise that is twofold: in the first glance, the meaning of the symbols is abstract, that is when the knitter initially looks at the chart and only recognizes it as a knitting chart but without a more specific meaning. The second situation can be that a knitter who already has a certain kind of experience with a knitting pattern would be able to already pre-determine what the chart contains. For example, someone might be able to tell, when looking at a chart, that it is for lace knitting or for a cabled pattern. Unless it is an extremely simple chart that only contains the basic stitches, such as knit and purl, most knitters will need to see the chart key to see the meaning of the symbols used. As long as the knitter does not know the chart in its smaller units - signs, it is still an image that hides a large amount of potentially complex specific language.

W. J. T. Mitchell highlights a problem that still exists today, as images (or symbols) can give a view into a new world. For a non-knitter, a knitting chart means nothing. It is just an image of an arrangement of symbols that might have been arranged for whatever reason, perhaps to create artistic work. Mitchell also (Mitchell, 1987, p. 9) notes that 'Images are not just a particular kind of sign, but something like an actor on the historical stage, a presence or Character endowed with legendary status". Every symbol used in a knitting chart is a character that acts according to its purpose. While it may seem, perhaps, a philosophical stance in a linguistic matter, it has a correlation with the fact that it is not just a sign, it is an actor that leads to the production of action.

There are no words in a chart symbol, but knowing what the symbol means, the reader can then decipher the action.

"That is the basic idea behind the concept of the language game: Whether language plays the central role or some more or less apparent role, our semiotic practices can be thought of as rule-guided practices. They are not chance actions nor randomly proffered words, but actions that owe their legitimacy, relevance and even existence to a set of rules determining their use." (Xanthos 2006).

What could be deemed a rule-guided practice when it comes to knitting? The first step is that the knitter looks at the chart carefully, determining what facts are clear from the start. Typically, nobody would start knitting a pattern without re-checking the key or explaining each symbol's meaning to a corresponding action to ensure that the instruction is fully understood.

The second step would be to search for the key to the chart. While some standard symbols are suggested to be used by the Craft Yarn Council (Knit Chart symbols n.d.). They are not obligatory as these are not standards regulated by ISO or DIN, so they are a mere suggestion, not a must. Many choose to use them, but no knitter can be sure whether the familiar-looking symbol means what they suspected it does.

Any designer or author of a knitting pattern is allowed to create their own symbols, especially if they use unusual stitch combinations that might not be known previously or obscure.

So, in this rule bound universe we have already determined two rules that need to be observed for successful comprehension of the chart:

1) An overall perusal of the chart

2) Reading of the chart key 
These might seem like trivial steps, but it is the rule that governs the signs if the chart is needed for practical purposes - to knit something based on it. There is, of course, also the option to ignore it all and observe the chart as a curiosity without any action plan.

After the two steps are implemented, the third is the process of memorizing what each symbol means in practical terms. It is not a mandatory rule as it is possible to always look back at the chart key to remember, but many readers would remember the meanings of these symbols after a while, especially if there is a repeat (Latvian - raports) - a set of stitches that can be repeated several times during the knitting process. For example, the entire pattern might have a large number of stitches that can reach several hundred, but the repeat represents, say, 20 stitches, so the knitter knows already that the symbols used in the chart apply to the entire work not just the 20 . This way, a large efficiency is possible, as a complex pattern can be shown on one page, whereas if it were written, it would take up dozens of pages as for a chart, there is no need to explain it stitch by stitch.

How do the creators of knitting charts explain them? It is important to also look at the issue from the perspective of a person who creates these charts.

Knitting pattern designer Emily Wessel (Wessel 2014) describes charts in this way: [the charts] are graphic representations of knitting instructions. They are a compact way to illustrate more patterns that would take more space if described in text instructions. Charts also illustrate how a lace, colorwork, or cable pattern will look once it is knit up, and this means that when you use charts, it is easier to see where you are in a pattern and identify errors early.

Wessel's definition of a charted pattern is quite similar to the author's opinion of this paper. Wessel, however, focuses more on the compactness and space than the meaning of the symbols within. She also dwells into subjective categories, such as her belief that a chart can show how the knitting will look when it is done. Her experience perhaps colours this as a knitting pattern designer - a person who has a done a lot of knitting will be able to picture the look of the final product. A novice at knitting might not. However, it does not exclude the novice from being able to read the chart and knit the pattern as paramount here is the sign, not the previous knowledge. If one knows the key to the chart, there are vast opportunities to learn new stitches not just by name but find audio-visual guides to them.

One tradition that can be traced back into the history of Latvian knitting. Books like Grasmane's (2021a) "Latvieša cimdi" and its translation "Mittens of Latvia" (Grasmane 2021b) which has had several editions through the years and is one of the most recognized sources on Latvian tradition knitting, rely heavily on the usage of charts. For each mitten, a colour chart represents each stitch, so anyone can use these patterns if they want to, as words are not the predominant guide in these texts. The colour chart, with each small square representing one stitch and one colour make it universally readable. It eliminates cultural aspects of pattern style and terminology, as there is no written text needed, just the symbols and colours, so no language or cultural perception can change that.

It leads back to the theory that a sign per semiotics can be anything, as discussed previously, a symbol used in a knitting chart could be a "/"or "- " without any specific reasoning behind its use. Indeed, "....within semiotics, representation is central but not primary, because the sign is not what represents so much as it is what causes the representation to be a representation of something other than itself." (Deely, 2009, p. 124). A red square might not represent anything much by itself, but in a traditional Latvian mitten, it is one of the keys to achieving knitting of the mitten itself.

Equally, a "/" by itself is nothing, but a knitting pattern represents the action "knit two stitches together". There are variations of the symbol possible, but it is still possible to grasp meaning with the chart key explanations.

In the classical model per Saussure, a sign represents a dyadic model. It is divided as the signifier (the sign vehicle) leads to the signifier (a conceptual or abstract content). This is, of course, a simplified and general approach to Saussure's theory, but the approach is straightforward, though sometimes it can be reasonably criticized (see Carassco 2014 for a different look at Saussure's work). In the situation of knitting charts, the graphic symbol used that represents the sign vehicle and the signifier is both the graspable concept - the visual perception of the stitch in the knit fabric and the abstract notion about what has to be done. The question remains whether this is the right model to look at the sign and its meaning as it is not just a straightforward process as the sign is in constant interaction with the user of the chart. 
Every separate sign used is an expression towards another; for example, the next two stitches need to be knitted together to reduce the overall stitch count because in the previous stitch, a yarn over, that is, an additional stitch was created. Without the previous symbol, the next one would not have a meaning, just as all symbols used previously and later in the same chart. It is a form of so called "unlimited semiosis". According to Novoselov (2014, p. 8) "Semiosis explains itself: This constant circular motion is a normal condition of signification, and it (this circular motion) even allows to use signs to speak about subjects and world conditions on a course of processes of communications". Unlimited semiosis also goes back to the previously mentioned repeat - the meanings constantly return to the knitter, it is a never-ending circle.

\section{Translation of knitting charts}

The description of the charted pattern and semiotics of the symbols used within might look like it excludes the need for translation. But the translator's role is incredibly important in the translation of the chart key. While the symbols, when together in a chart, do not need a word for word translation of their meaning, they need a translation of each symbol used. The translator's work might seem relatively short, as it is only needed to translate the given symbols, but the text itself needs to be correct and easily understandable. Unlike in a longer text, where the translator themselves might notice a discrepancy in their translation and the correct meaning and return to passages to correct the mistake, a translation for a chart is much shorter and can be likely to contain mistakes if the translator is not familiar with the specifics of charted knitting patterns and their usage. "Like the technical writer, the translator uses information from a variety of sources, not just the source text, to produce a target text which is effective, and which performs the desired communicative function." (Byrne 2006, 15)

An advantage would be if the translator has previous knitting experience and knows what to expect from a charted knitting pattern. The specifics of the entire translation project also impact the end result; for example, is it a book that is devoted to charted knitting stitch patterns, is it a single knitting pattern that needs to be translated for a magazine. The complexity of the translation process increases the larger the amount of individual, unique charts is; more charts lead to more separate symbols. Each symbol must be given equal consideration for correct and careful translation as it is the translator's and publisher's chance to show that their product is suitable for the end-user.

What is important in the translation of the symbol:

- Conciseness

- Precision

- Vocabulary used

The caveat in the translation process is that the original English publications tend to use the typical abbreviated style of the written pattern instruction and explain the symbols. For example, "l" can be described as ktbl on RS, ptbl on WS (in the original of titled The knitter's stitch collection (Stanfield\&Griffiths 2010) as the book gives also written instructions for some (but not all) patterns listed in the collection). What does this abbreviate? If the knitter is familiar with the abbreviated forms, they are likely to decipher it as "Knit through back loop on the right side of the work, purl through the back loop of the wrong side of the work". Here we see a double economy. The symbol saves the space of the longer explanation and creates a double economy of wording in the terms that it describes actions both on the right (visible) side of the work and the wrong (hidden) side of the work.

The Latvian translation of the aforementioned title, that was published in 2015 (Stenfilda \& Grifitsa 2015), Latvian translation text economizes textual volume even further, translating the English $k t b l$ and $p t b l$ as $l g r-L P, k g r-K P$, making it even shorter character-wise and making the reader to do more "work" to find the meaning of the abbreviation. As the reader has to be able to piece together that $\lg r$ is a combination of labiski+griezti; similarly, $k g r$ is kreiliski+griezti. As this is a translation for the meaning of a certain symbol is used through the original English book and also the Latvian translation of it, such economy should be used with utmost care.

These are relatively easy stitch combinations, and their abbreviated state is potentially guessable based on the list of abbreviations given in the translated book and the reader's previous knowledge. 
Perhaps this was also done in the hopes that it would avoid all long sentences and descriptions, creating long dependencies of text, that a too-long description of a symbol would confuse the reader and thus impact the communication of the reader and text.

If language users are motivated by avoiding difficulty, then they should avoid long dependencies. Furthermore, if languages have evolved to support easy communication, then they should not enforce word orders that create long dependencies. (Futrell et al., 2015, 10335).

With an economic abbreviation of a symbol, there is, in theory, less potential for confusion, if the reader already knows what $\lg r$ means, then there is no risk of misunderstanding. But it seems like a possibly bold assumption of the translator that the reader will understand the abbreviations and the efforts to make it all more efficient space-wise. There still remains a chance that the reader cannot figure out the meaning of the abbreviations used based on the list.

Korkas and Pavlidis (2004) state that "it is generally accepted that, when dealing with specialist texts, the translator needs at least a working knowledge of the domain or subject-field concerned, i.e., familiarity with the underlying concepts and constructs, and their linguistic interpretation, preferably in both source and target language, thus ensuring the use of accurate terminology and stylistically appropriate phraseology, which comprises the LSP."

It can be said that these translators that translate knitting books published by reputable publishers for a wide audience possess the needed background knowledge, so it might be the reason why some translations take some 'liberties' within the translation content, like the aforementioned introduction of abbreviations in chart symbol translation and attempts at economizing space. They might think that all will be clear to the reader as it is clear to them (the translator), but it might not be so. Readers might not intuitively find the correct symbol and its meaning. Therefore, another intermediary between the original text and its translation would be needed - a skilled technical editor who has a reasonably good understanding of both knitting cultures represented in the text (that is, the source and the target) and knows what readers expect. While readers are often open to learning new concepts, especially in creative pursuits such as knitting, they might abandon a certain publication if it is unclear to them.

\section{Conclusions}

Can charted patterns be universals for any language? Symbols could be the solution for transmitting knitting traditions of different cultures globally as only very little text is needed to accompany the knitting chart. It depends on the translators' background knowledge and specific understanding of the symbols used in a chart, but if the translation is done well, the readers should be able to easily replicate all charts.

Thus, massive savings of space and text can be achieved as unlike in written patterns where each stitch, each action needs to be described in detail, there is no need to write out everything. If a knitting stitch pattern has a chart, then it can be made available to any readers who wish to try it, for example, recently more international knitters are interested in Japanese charts. When the chart key is translated, these charts and readers know what each symbol means to break the barrier between East and West.

This also shows the untapped perspectives in linguistics and translation studies research when it comes to specialised niche texts that have not yet been looked at in previous research. These texts, which are perhaps unusual to the general reading public, show that language and symbol used in real-life applications can differ from theoretical perceptions and introduce new understandings.

\section{Bibliography}

Byrne, J. (2006). Technical translation: Usability strategies for translating technical documentation. Spinger.

Carrasco, B. (2014). From the Sign to the Passage: A Saussurean Perspective. Semiotics, 555-574.

Danesi, M. (2004). Messages, signs, and meanings: A basic textbook in semiotics and communication (Vol. 1). Canadian Scholars' Press.

Deely, J. (2009). Purely objective reality (Vol. 4). Walter de Gruyter.

Futrell, R., Mahowald, K., \& Gibson, E. (2015). Large-scale evidence of dependency length minimization in 37 languages. Proceedings of the National Academy of Sciences, 112(33), 10336-10341.

Grasmane, M. (2021a). Latvieša cimdi (3rd ed.). [Latvian Mittens].Senā Klēts.

Grasmane, M. (2021b). Mittens of Latvia: 178 Traditional Designs to Knit (1st ed.). National Costume Center Sena Klets.

Korkas, V., \& Pavlides, P. (2004). Teaching aspects of LSP (Language for Special Purposes) to non-specialists: A case for background courses in translation studies programmes. JoSTrans-The Journal of Specialised Translation, 2. 
Knit Chart Symbols. (n.d.). Craft Yarn Council. https://www.craftyarncouncil.com/standards/knit-chart-symbols Stanfield, L., \& Griffiths, M. (2010). The Essential Stitch Collection: A Creative Guide to the 300 Stitches Every Knitter Really Needs to Know (1st edition). Readers Digest.

Stenfîlda, L.\&Grifitsa, M. (2015). Adījumu rakstu kolekcija. [Collection of knitting stitches].Zvaigzne ABC

Symbol. (2021). Merriam-Webster. https://www.merriam-webster.com/dictionary/symbol

Mitchell, W. T. (1987). Iconology: image, text, ideology. University of Chicago Press.

Novoselov, V. (2014). Meaning, interpretation and unlimited semiosis. Social Sciences, 3(1), 5-8.

Wessel, E. (2014). How to read a knitting chart. Tin Can Knits. https://blog.tincanknits.com/2014/06/06/how-to-read-aknitting-chart

Xanthos, N. (2006). Wittgenstein's Language Games. Signosemio. http://www.signosemio.com/wittgenstein/languagegames.asp. 